\title{
Transfer RNA detection by small RNA deep sequencing and disease association with myelodysplastic syndromes
}

Yan Guo ${ }^{1}$, Amma Bosompem², Sanjay Mohan ${ }^{4}$, Begum Erdogan², Fei Ye ${ }^{3}$, Kasey C. Vickers ${ }^{4}$, Quanhu Sheng ${ }^{1}$, Shilin Zhao', Chung-I Li ${ }^{5}$, Pei-Fang Su', Madan Jagasia ${ }^{4}$, Stephen A. Strickland ${ }^{4}$, Elizabeth A. Griffiths ${ }^{7}$ and Annette S. Kim ${ }^{2,8^{*}}$ (D)

\begin{abstract}
Background: Although advances in sequencing technologies have popularized the use of microRNA (miRNA) sequencing (miRNA-seq) for the quantification of miRNA expression, questions remain concerning the optimal methodologies for analysis and utilization of the data. The construction of a miRNA sequencing library selects RNA by length rather than type. However, as we have previously described, miRNAs represent only a subset of the species obtained by size selection. Consequently, the libraries obtained for miRNA sequencing also contain a variety of additional species of small RNAs. This study looks at the prevalence of these other species obtained from bone marrow aspirate specimens and explores the predictive value of these small RNAs in the determination of response to therapy in myelodysplastic syndromes (MDS).

Methods: Paired pre and post treatment bone marrow aspirate specimens were obtained from patients with MDS who were treated with either azacytidine or decitabine (24 pre-treatment specimens, 23 post-treatment specimens) with 22 additional non-MDS control specimens. Total RNA was extracted from these specimens and submitted for next generation sequencing after an additional size exclusion step to enrich for small RNAs. The species of small RNAs were enumerated, single nucleotide variants (SNVs) identified, and finally the differential expression of tRNA-derived species (tDRs) in the specimens correlated with diseasestatus and response to therapy.

Results: Using miRNA sequencing data generated from bone marrow aspirate samples of patients with known MDS $(N=47)$ and controls $(N=23)$, we demonstrated that transfer RNA (tRNA) fragments (specifically tRNA halves, tRHs) are one of the most common species of small RNA isolated from size selection. Using tRNA expression values extracted from miRNA sequencing data, we identified six tRNA fragments that are differentially expressed between MDS and normal samples. Using the elastic net method, we identified four tRNAs-derived small RNAs (tDRs) that together can explain $67 \%$ of the variation in treatment response for MDS patients. Similar analysis of specifically mitochondrial tDRs (mt-tDRs) identified $13 \mathrm{mt}-t$ Rs which distinguished disease status in the samples and a single mt-tDR which predited response. Finally, 14 SNVs within the tDRs were found in at least $20 \%$ of the MDS samples and were not observed in any of the control specimens.

(Continued on next page)
\end{abstract}

\footnotetext{
* Correspondence: akim@partners.org

${ }^{2}$ Department of Pathology, Immunology, and Microbiology, Vanderbilt

University Medical Center, Nashville, TN, USA

${ }^{8}$ Present address: Brigham and Women's Hospital, 75 Francis Street, Boston,

MA 02115, USA

Full list of author information is available at the end of the article
}

\section{Biomed Central}

(c) 2015 Guo et al. Open Access This article is distributed under the terms of the Creative Commons Attribution 4.0 International License (http://creativecommons.org/licenses/by/4.0/, which permits unrestricted use, distribution, and reproduction in any medium, provided you give appropriate credit to the original author(s) and the source, provide a link to the Creative Commons license, and indicate if changes were made. The Creative Commons Public Domain Dedication waiver (http://creativecommons.org/publicdomain/zero/1.0/) applies to the data made available in this article, unless otherwise stated. 
(Continued from previous page)

Discussion: This study highlights the prevalence of tDRs in RNA-seq studies focused on small RNAs. The potential etiologies of these species, both technical and biologic, are discussed as well as important challenges in the interpretation of tDR data.

Conclusions: Our analysis results suggest that tRNA fragments can be accurately detected through miRNA sequencing data and that the expression of these species may be useful in the diagnosis of MDS and the prediction of response to therapy.

Keyword: Transfer RNA (tRNA), microRNA (miRNA), Next generation (deep) sequencing (NGS), Predictive biomarkers, DNA methyltransferase inhibitor, Response to therapy, Myelodysplastic syndromes (MDS)

\section{Background}

RNA next generation sequencing (RNA-seq) technology has replaced microarrays as the primary platform for gene expression profiling [1-4]. This same technology has also been used to study the expression of microRNAs (miRNAs), although there are fewer direct comparisons between miRNA arrays and miRNA-seq [5-7]. During miRNA-seq library preparation, small RNAs are selected by electrophoresis on a size typically ranging from 20 to 50 nucleotides (nts). This range of size selection allows for the capture of many other species of small RNAs in addition to miRNAs [8-15].

One of the major byproducts of size selection for miRNAs is tRNA-derived small RNAs (tDRs) $[13,16]$. This may be the result of either active cleavage or an artifact of the miRNA-seq library construction. Full length or parent tRNAs are adaptor molecules composed of RNA with a length typically ranging from 73 to 94 nts. They serve as the physical link that translates messenger RNA into protein. Cleavage of tRNAs by the RNAse III enzyme, angiogenin, may occur in a number of reactive conditions to produce tRNA-derived halves (tRHs) [17, 18]. Likewise, tRNAs can also be cleaved in a Dicer-dependent manner or as an in vitro phenomenon by incubation with $\mathrm{MgCl}_{2}$ or nuclease S1 [10, 19]. All of these processes result in cleavage within a hairpin loop. D loop cleavage results in a 19 nt tRNA-derived fragment (tRF), and cleavage of the anticodon loop before the anti-codon affords a $33 \mathrm{nt} \mathrm{tRH}$ $[10,19]$. These 5 ' tDR products, in particular the 33-base tRH, may be captured by miRNA-seq.

In addition, apparent tDRs and tRNAs with base errors found in RNA-seq datasets may arise from the extensive chemical modifications of parent tRNA nucleotides. While these modifications contribute physiologically to RNA stability, function, and structure [20], they may be either misincorporated during the requisite reverse transcription step [21], resulting in specific base errors [22], or result in blockage of reverse transcription, with production of a truncated cDNA product [23]. Therefore, sequencing datasets may contain many reads that appear to be tDRs, but in fact are actually derived from full-length parent tRNA templates.

The quantification of tRNAs and tDRs warrant study through high-throughput sequencing. Aberrations in tRNAs have been linked to a range of diseases, including neurological disorders [24-26], cancer [27-29], type II diabetes [30-32], and mitochondrial diseases [33-35]. In addition, $\mathrm{tDRs}$ have been identified in a number of cancer cell lines, and some tDRs may play defined biological roles, such as the role of tRF-1001 in proliferation [11]. Thus far, only one study has found a link between somatic mutations in mitochondrial tRNA (Leu (UUR), aka MT-TL1) and myelodysplastic syndromes (MDS) [36]. The potential functionality of tRNA in MDS remains largely unexplored.

As part of our efforts to study MDS, we have previously examined miRNA expression in MDS $[37,38]$. MDS is a common disease of the elderly (median age 71-76) [39] characterized by ineffective maturation of hematopoietic cells. This ineffective maturation manifests clinically as low blood counts and morphologically as dysplasia. The MDS state has been associated with expression changes in miRNAs. Several entities have been discovered on 5q, which contribute to the disease phenotype in the $5 \mathrm{q}$ minus syndrome subtype of MDS [40]. miR-145 and miR146 have recently been identified in the commonly deleted region, and their knockdown appears to be associated with the thrombocytosis and dysmegakaryopoiesis seen in $5 \mathrm{q}$ minus syndrome [41]. Researchers, including those in our group, have identified several other miRNAs whose expression is dysregulated with the diagnosis of other subtypes of MDS [37, 38, 42-45]. Published research on other types of small RNAs in MDS is limited.

MDS has a median survival of only 18-24 months [46], with death resulting from either cytopenic complications or transformation to acute myeloid leukemia (AML) [47]. Currently, there are only two FDA-approved drugs for the treatment of all types of MDS, both of which are DNA methyltransferase inhibitors (DNMTIs): 5-azacytidine, and decitabine. However, only $40-47 \%$ of patients achieve 
the clinically meaningful responses of hematologic improvement or better with DNMTIs [48]. Although several recent studies have suggested the use of mutational analysis by DNA next generation sequencing (NGS, DNAseq) [49], genetics [50], or the presence or absence of key pharmacodynamic and pharmacokinetic markers [51] to predict response, as yet, there are no clinical or laboratory parameters in routine clinical practice that accurately predict response to DNMTIs [52-54]. The methylation status of specific loci has been shown to be prognostically relevant in the treatment of a closely related disease, chronic myelomonocytic leukemia, by decitabine [55].

In this study, we examined the expression of tRNAs in paired pre- and post-treatment samples from patients with the diagnosis of MDS on receiving treatment with DNMTIs. In miRNA sequencing data, the abundance of tRNA fragments vastly outnumbers miRNAs. This gives us a great opportunity to study tRNA expression using miRNA sequencing data through their anticodon sequences.

\section{Methods}

\section{Ethics statement}

This study was conducted in accordance with the Declaration of Helsinki with the approval of the Institutional Review Boards at Roswell Park Cancer Institute and Vanderbilt University Medical Center.

\section{Consent statement}

Written informed consent for the patient-derived specimens was obtained prior to the acquisition in all cases. The consents were approved by the Institutional Review Boards at Roswell Park Cancer Institute and Vanderbilt University Medical Center.

\section{Specimen description}

Bone marrow aspirate specimens $(N=69)$ (see Additional file 1: Table S1) were obtained from the Roswell Park Cancer Institute with appropriate approval by the Institutional Review Boards at Roswell Park and Vanderbilt University Medical Center. Twenty-two of the 69 bone marrow samples were from control patients (staging marrows that were negative for hematolymphoid malignancy). The other 47 samples were from MDS patients. Out of 47 MDS samples, 24 were pre-treatment specimens, and 23 were obtained after treatment with 5azacytidine or decitabine, both DNMTIs. For each of the pre- and post-treatment paired samples, a response score (range: 1-6, where 1 represents complete remission, 2 complete marrow remission, 3 partial remission, 4 hematologic improvement, 5 stable disease, and 6 progressive disease) was obtained based on clinical and pathological criteria to indicate the effectiveness of treatment (Additional file 1: Table S1) [56]. Bone marrow mononuclear cells (BM-MNCs) were isolated from fresh, unsorted bone marrow aspirate specimens $(N=69)$ by the Ficoll method using Cellgro Lymphocyte Separation Medium (Corning, Manassas, VA). After performing a cell count, the cells were re-pelleted from Dulbecco's phosphate buffered saline solution and resuspended in Gibco Recovery Freezing medium (Invitrogen, Grand Island, $\mathrm{NY}$ ), and then frozen gradually, prior to storage in liquid nitrogen. Cells were frozen at a density of 10 to 20 million cells per $\mathrm{mL}$.

\section{RNA isolation and RNA sequencing}

Total RNA (totRNA) was isolated using a mirVana miRNA isolation kit (Life Technologies, Grand Island, NY) per manufacturer's instructions. Sequencing library construction was performed on the totRNA from all 69 samples, each obtained from a single bone marrow aspirate specimen. RNAs were isolated by the mirVana RNA isolation kit, which was found to perform better than the TRIzol miRNA isolation kit [13]. Libraries were prepared using the TruSeq Small RNA sample preparation kit (Illumina, San Diego, CA). The small RNA protocol specifically ligates RNA adapters to mature miRNAs that have a 5'-phosphate and 3'-hydroxyl group resulting from enzymatic cleavage by RNA processing enzymes like Dicer. In the first step, RNA adapters were ligated onto each end of the RNA molecules, and a reverse transcription reaction was used to create singlestranded cDNA. This cDNA was then PCR amplified with a universal primer and a second primer containing one of 48 uniquely indexed tags to allow multiplexing. Size selection of the cDNA constructs was performed using a $3 \%$ gel cassette on the Pippin Prep (Sage Sciences, Beverly, MA) to reduce the library to mature miRNAs and other regulatory RNAs in the 20-30 bp size range and to remove adapter-adapter products. The resulting cDNA libraries then underwent a quality check on the Bioanalyzer HS DNA assay (Agilent, Santa Clara, CA) to confirm the final library size and on the Agilent Mx3005P quantitative PCR machine using the KAPA library quantification kit (Illumina, San Diego, CA) to determine concentration. A $2 \mathrm{nM}$ stock was created, and samples were pooled by molarity for multiplexing. From the pool, $10 \mathrm{pM}$ was loaded into each well for the flow cell on the Illumina cBot for cluster generation. The flow cell was then loaded onto the Illumina HiSeq 2500 utilizing v3 chemistry and HTA 1.8. The raw sequencing reads in BCL format were processed through CASAVA1.8.2 for FASTQ conversion and demultiplexing. The RTA chastity filter was used, and only the PF (pass filter) reads were retained for further analysis.

\section{Sequencing data analysis}

We implemented a custom analysis pipeline for small RNA sequencing data processing. We used Cutadapt 
[57] to trim 3' adapters for raw reads. Quality control on raw data was performed using QC3 [58]. All reads with length less than 12 were discarded. The adaptortrimmed reads were formatted into a non-redundant FASTQ file where the read sequence and copy number was recorded for each unique tag. The usable unique reads were mapped to the whole genome using Bowtie1 [59] with only one mismatch allowed. The latest Sanger microRNA database, miRBase20 [60], was used to quantify microRNA isomiRs by reads mapped with position $0,+1$, and +2 from the 3 ' terminal of the miRBase locus. The tRNA database was prepared by combining the latest UCSC TRNA database with the tRNA loci of mitochondria from the ensembl database [61]. The reads mapped with tRNA loci were used not only for tRNA quantification, but also for tRNA mapping position coverage analysis. The reads mapped to miRNAs, tRNAs, and other small RNAs (including lincRNAs, snoRNAs, snRNAs, rRNAs, and misc_RNAs in the ensembl database) were used for response category analysis. In addition, single nucleotide variations (SNVs) can also be detected through RNA-seq methods [14, 62-64]. We identified SNVs in the tRNA reads using VarScan 2 [65] and filtered the SNVs based on the VarScan 2 recommended filters. Inconsistent SNVs between paired samples were removed from analysis. Only SNVs with zero appearance in control samples were included in the analysis.

\section{Response analysis}

A linear regression model was fit using response scores as outcomes and tRNA expression as predictors. Differential expression between MDS biopsy samples and control samples were conducted using MultiRankSeq [66], which is based on the combination of results from three distinct RNA-seq analysis packages: DESeq [67], edgeR [68], and baySeq [69]. A tRNA was considered significantly differentially expressed if the adjusted p-values for all three analysis packages were less than 0.05 . The elastic net method [70] was used to select a panel of tRNAs that together explain a large proportion of the variation in patient response. Elastic net regularization [71] is a technique that conbines the L1 and L2 penalties of the lasso and ridge regression methods. The elastic net method provides variable selection to produce parsimonious and interpretable models in the $p>>N$ case without being severely limited by the sample size. This method greatly improves performance in the case of highly correlated predictor variables (as we expect to have in clinical data), through the identification of groups of phenotypes with significantly high correlation that contribute the most to the variation in the data. Unsupervised hierarchical cluster analysis was conducted using HeatMap3 [72]. Functional analysis of tRNAs/tDRs was carried out manually by searching online due to the lack of a functional database for tRNA. In addition, a Fisher's exact test was used to identify SNVs associated with MDS.

\section{Results}

We found that for the reads obtained using a standard miRNA-seq protocol, the number of reads aligned to tDRs (78.81 \% of reads) vastly outnumbered those aligning to miRNAs (4.43\% of reads) (Fig. 1a). The read length distribution, after trimming the adaptor with Cutadapt [57], shows a strong modal peak at 33 nucleotides, with the expected 22 nucleotide mean length of miRNAs (Fig. 1b)
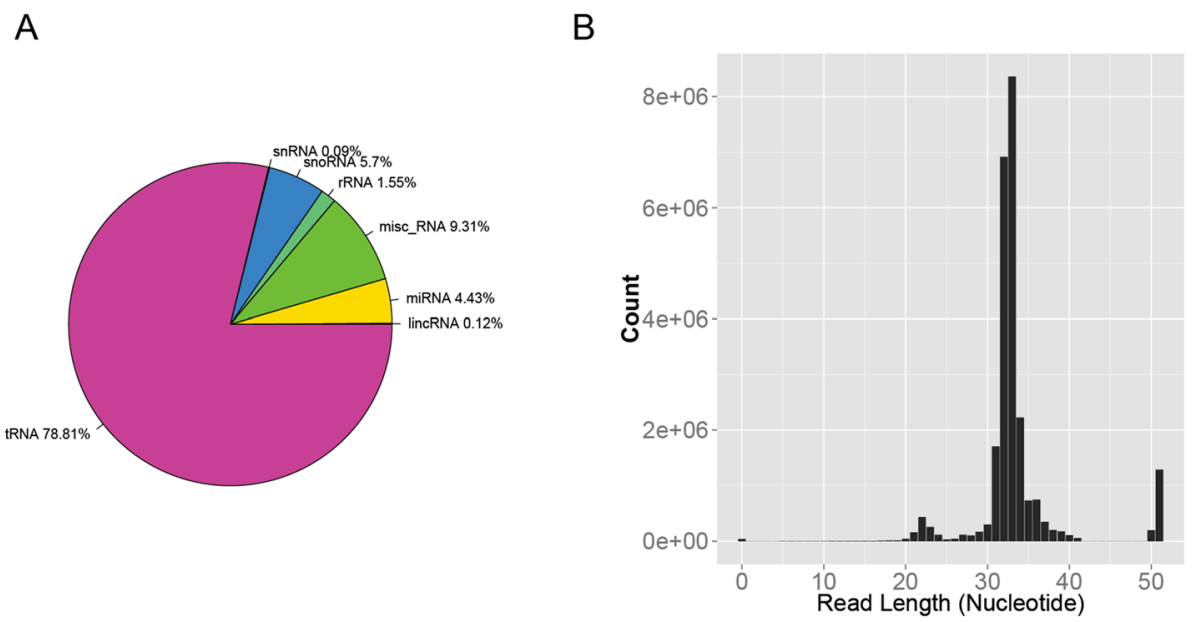

Fig. 1 Read count and alignment distribution example taken from one sample. The figures were produced using all read counts per category, not just unique reads per category. The other samples in this study follow a similar pattern. a. Read count distribution after trimming adaptors. The smaller peak at 22 base pairs indicates the abundance of miRNA and the larger peak at 33 base pairs indicates the abundance primarily of tRNA. $\mathbf{b}$. The reads alignment distribution by RNA type. The majority of the reads aligned to tRNA instead of miRNA 
Table 1 Differentially expressed tRNA derivatives (MDS versus control samples)

\begin{tabular}{lrlccc}
\hline tRNA & $\begin{array}{r}\log ^{2} \text { FCC }^{\mathrm{a}} \\
\text { DESeq2 }\end{array}$ & $\begin{array}{l}\text { pAdj }^{\mathrm{b}} \\
\text { DESeq22 }\end{array}$ & $\begin{array}{l}\text { log2FC } \\
\text { edgeR }\end{array}$ & $\begin{array}{l}\text { pAdj } \\
\text { edgeR }\end{array}$ & $\begin{array}{l}\text { pAdj } \\
\text { baySeq }^{c}\end{array}$ \\
\hline chrM.tRNA10-TC & 1.2840 & 0.0006 & 2.5347 & 0.0005 & 0.0011 \\
chr12.tRNA8-AlaTGC & 0.8518 & 0.0034 & 1.9498 & 0.0005 & 0.0378 \\
chr16.tRNA4-ProAGG & 0.8072 & 0.0274 & 1.6531 & 0.0043 & 0.0089 \\
chr1.tRNA58-LeuCAA & -1.0706 & 0.0000 & -0.7228 & 0.0406 & 0.0000 \\
chr19.tRNA8-SeC(e)TCA & -0.6461 & 0.0103 & -0.5944 & 0.0289 & 0.0126 \\
chr19.RRNA4-ThrAGT & -0.8098 & 0.0067 & -0.7906 & 0.0403 & 0.0489 \\
\hline
\end{tabular}

${ }^{a} \log 2 \mathrm{FC}=\log 2$ fold change

${ }^{b}$ pAdj $=$ adjusted $\mathrm{p}$-value

'DESeq2, edgeR, and baySeq are the three RNAseq differential expression analysis packages used in this analysis. BaySeq does not generate fold change, thus no fold change from bayseq can be reported

forming only a small secondary peak in the distribution. The peak at 33 bases indicates the abundance of tRNA species that have been cleaved in the anticodon loop from their full length of 73-94 nucleotides [10, 19]. The complete sequenced position of each tRNA is provided in Additional file 2: Figure S1. Numerous small RNA mapping statistics can be located in (Additional file 1: Table S2) (Fig. 1b).

Using MultiRankSeq, we identified six tDRs that are significantly differentially expressed between MDS samples and control samples (Table 1, Fig. 2). Three tDRs demonstrated increased expression in MDS (chrM.tRNA10.TC, chr12.tRNA8.AlaTGC, and chr16.tRNA4.ProAGG) while three were decreased (chr1.tRNA58-LeuCAA, chr19.tR NA8-SeC(e)TCA (SeC(e)TCA), and chr19.tRNA4-ThrA GT). The complete results can be viewed in Additional file 1: Table S3. Unsupervised cluster analysis showed that tRNA profiling was able to distinguish between MDS and control samples $\left(\chi^{2} p<0.0001\right)$. No tDRs were significantly differentially expressed between pre- and posttreatment samples (Additional file 1: Table S4, Fig. 2).

Using the elastic net with ten-fold cross-validation (CV) for selecting the model with the smallest CV error, we identified a panel of four tRNA fragments (chr6.tRN A157.ValCAC, chr11.tRNA17.ValTAC, chrM.tRNA12.TS1, and chrX.tRNA4.ValTAC) whose combined expression in the pre-treatment samples together are predictive of the likelihood of response. By fitting a multivariable linear regression model using expression values of these four tDRs, two out of the four tRNA species showed significant associations with response (chr6.tRNA157.ValCAC $p=$ 0.03564 and chrM.tRNA12.TS1 $p=0.01362$ ) adjusted for other variables in the model. Overall, the model explained roughly $67 \%$ of the variation in treatment response $\left(R^{2}=\right.$ 0.6735) (Table 2). It should be noted that the first $33 \mathrm{nu}-$ cleotides of chr11.tRNA17.ValTAC and chrX.tRNA4.Val TAC show perfect sequence homology, so these different tRNAs cannot be distinguished by these methods. Therefore, it is possible that one or the other species are predictive of response rather than both.

Using the change of tDR expression between pre- and post-treatment (computed as percentage of change), we

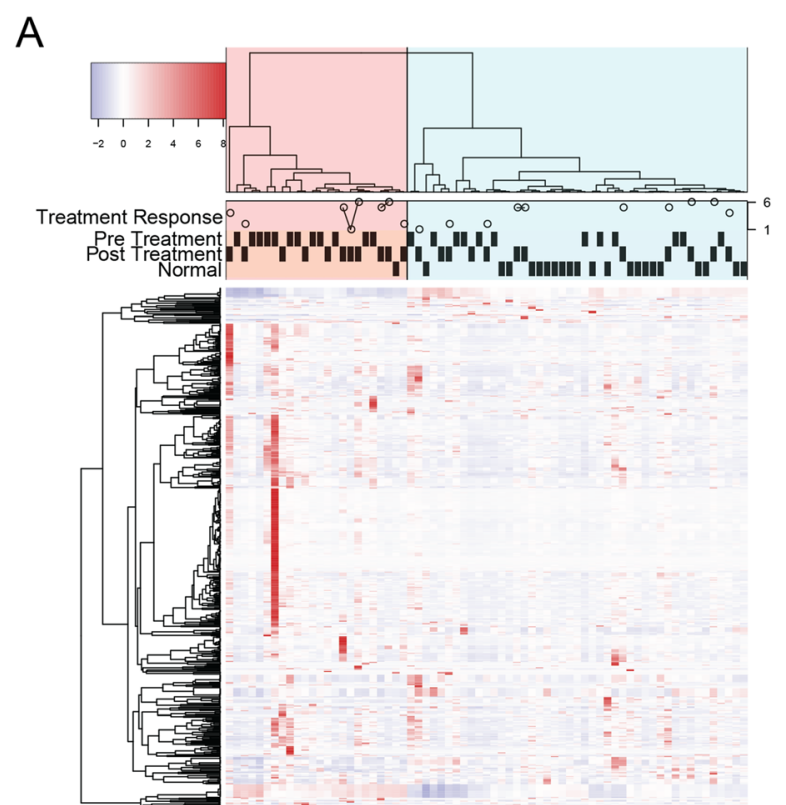

\section{B}
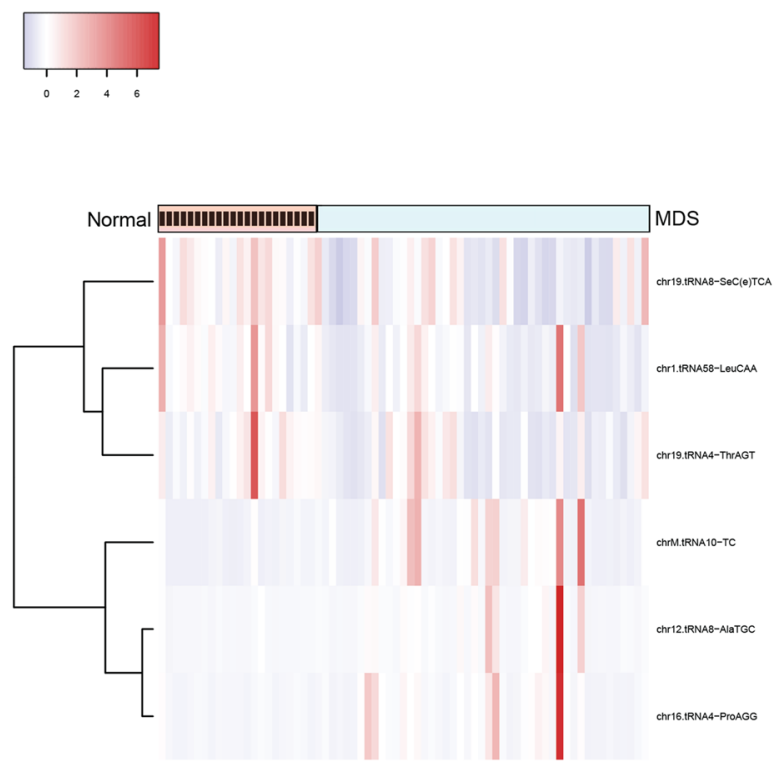

Fig. 2 a. Cluster analysis and heatmap using tRNA expression of all samples. Three phenotype bars are drawn below the dendrogram: pretreatment, post-treatment and normal controls. Two clusters are visible (light green and light red). These two clusters do not separate pre- and post-treatment, but distinguish MDS and normal samples reasonably well. b. The six differentially expressed tRNA between disease and normal 
Table 2 Linear regression results on tRNAs and treatment association

\begin{tabular}{llrl}
\hline & tRNA & Effect $^{a}$ & p value \\
\hline Treatment & chr6.tRNA157.ValCAC & 0.0149 & 0.0356 \\
Response & chr11.tRNA17.ValTAC & 0.1415 & 0.8343 \\
R-squared $=0.67$ & chrM.tRNA12.TS1 & -0.4023 & 0.0136 \\
& chrX.tRNA4.ValTAC & -0.0058 & 0.9931 \\
Post vs Pre & chr1.tRNA35.GlyGCC & -12.9856 & 0.3134 \\
R-squared =0.40 & chr19.tRNA9.PseudoTT & 0.0038 & 0.0507 \\
& chr21.tRNA2.GlyGCC & 12.9798 & 0.3136
\end{tabular}

Notice that not all tRNA are significant in this table; however, acting together, they explain the greatest amount of variation in treatment

${ }^{a}$ Effect is explained as per 1 unit increase in tRNA expression; the treatment changes the effect amount of unit

conducted the same statistical analysis. Three tDRs (chr1.tRNA35.GlyGCC, chr21.tRNA2.GlyGCC, and chr1 9.tRNA9.PseudoTTT) were identified as predictors for treatment response. Multivariate linear regression was fit using treatment response as the outcome and tDR expression changes in the three tDRs as predictors. PseudoTTT trended towards an association with treatment response $(p=0.0507)$. Roughly $40 \%$ of the variation in patient responses can be explained using the difference of expression between pre- and post-treatment of these three tDRs $(\mathrm{R}$-squared $=0.4056)$ (Table 2). It should be noted that the first 33 nucleotides of chr1.tRNA35.GlyGCC and chr21 .tRNA2. GlyGCC are also homologous, leading to alignment ambiguity.

Functional correlation of tRNA expression is challenging due to the dearth of available public resources focused on tRNAs. We focused on mitochondrial tRNAs (mt-tRNAs) as a limited subset of tRNA species to study, in particular those mt-tRNAs that have a demonstrated disease association in the literature (see references listed in Table 3). In Table 3, we show the false discovery rate (FDR) p-values for the ability of these tDRs from differential expression analysis methods to distinguish between MDS and control (Table 3, columns 3-5) and their association with treatment response (Table 3, columns 6-8) for all $22 \mathrm{mt}-\mathrm{tDRs}$. Thirteen of the $22 \mathrm{mt}-\mathrm{tDRs}$ had significantly adjusted p-values from at least two of the three methods from MultiRankSeq in the discrimination of

Table 3 Test statistics of previously identified mitochondria tRNAs with disease associations

\begin{tabular}{|c|c|c|c|c|c|c|c|}
\hline \multirow[b]{2}{*}{ tRNA } & \multirow[b]{2}{*}{ Association } & \multicolumn{3}{|c|}{$\begin{array}{l}\text { Differential expression of tRNA MDS } \\
\text { vs control }\end{array}$} & \multicolumn{3}{|c|}{$\begin{array}{l}\text { Linear regression association of tRNA } \\
\text { with response }\end{array}$} \\
\hline & & pAdj (DESeq2) & pAdj (edgeR) & pAdj (baySeq) & Effect & Std err & $p$ value \\
\hline MT-TF & MELAS, MT-TF-related, MERRF syndrome [88] & 0.0103 & 0.0015 & 0.4869 & -1.3378 & 3.5754 & 0.7129 \\
\hline MT-TV & Leigh syndrome, NARP, mitochondrial disorder [89] & 0.0103 & 0.0020 & 0.6661 & -0.1755 & 0.2952 & 0.5602 \\
\hline MT-TL1 & myelodysplastic syndrome [39] & 0.0299 & 0.0030 & 0.4143 & 0.7728 & 1.1559 & 0.5127 \\
\hline MT-TI & hypertrophic cardiomyopathy [90] & 0.0431 & 0.0044 & 0.7070 & -1.3977 & 2.8147 & 0.6258 \\
\hline MT-TQ & MELAS [91] & 0.4745 & 0.0687 & 0.9095 & -0.1673 & 0.2261 & 0.4693 \\
\hline MT-TM & mitochondria disorder, hypertension [92] & NA & 0.0354 & 0.6065 & -0.6015 & 0.2818 & 0.0476 \\
\hline MT-TW & neonatal onset mito disease [93] & 0.0271 & 0.0040 & 0.4824 & -18.3065 & 27.0968 & 0.5084 \\
\hline MT-TA & hearing loss [94] & 0.0062 & 0.0015 & 0.2336 & -33.3062 & 19.0142 & 0.0979 \\
\hline MT-TN & Ophthalmoplegia [95] & 0.0134 & 0.0024 & 0.5159 & -6.6839 & 3.0347 & 0.0417 \\
\hline MT-TC & hearing loss [96] & 0.0006 & 0.0005 & 0.0011 & -0.7931 & 1.1525 & 0.5006 \\
\hline MT-TY & mitochondrial cytopathy [97] & 0.5648 & 0.0642 & 0.9019 & 0.0994 & 3.1135 & 0.9749 \\
\hline MT-TS1 & hearing loss [98] & 0.0017 & 0.0009 & 0.1043 & -54.1283 & 17.2749 & 0.0061 \\
\hline MT-TD & mitochondrial myopathy [99] & NA & 0.1135 & 0.8845 & -14.0023 & 9.2086 & 0.1467 \\
\hline MT-TK & MELAS [100] & 0.6851 & 0.1933 & 0.9098 & -0.4508 & 0.5028 & 0.3825 \\
\hline MT-TG & hypertrophic cardiomyopathy [101] & 0.0775 & 0.0067 & 0.7119 & 0.4488 & 4.0525 & 0.9131 \\
\hline MT-TR & mitochondria myopathy [102] & 0.4745 & 0.1346 & 0.8194 & -19.9639 & 10.4921 & 0.0742 \\
\hline MT-TH & MELAS [103] & 0.1634 & 0.0251 & 0.8843 & -0.4952 & 0.4202 & 0.2549 \\
\hline MT-TS2 & mitochondrial myopathy [104] & 0.0431 & 0.0043 & 0.8107 & -0.0547 & 0.2504 & 0.8295 \\
\hline MT-TL2 & ophthalmoplegia [105] & 0.3483 & 0.0534 & 0.9062 & -1.1874 & 1.2812 & 0.3670 \\
\hline MT-TE & myopathy [106] & 0.0271 & 0.0045 & 0.5381 & -0.3868 & 0.2411 & 0.1271 \\
\hline MT-TT & multiple sclerosis [107] & 0.3483 & 0.0466 & 0.8629 & -9.0631 & 11.8617 & 0.4553 \\
\hline MT-TP & mitochondrial catopathy [108] & 0.0034 & 0.0007 & 0.4374 & -4.2378 & 3.7122 & 0.2694 \\
\hline
\end{tabular}


Table 4 tRNA single nucleotide variant analysis

\begin{tabular}{|c|c|c|c|c|c|c|c|c|c|c|}
\hline tRNA & Known SNP & $\mathrm{CHR}$ & POS & REF & ALT & $\begin{array}{l}\text { Control } \\
\text { with SNV }\end{array}$ & $\begin{array}{l}\text { Control } \\
\text { without SNV }\end{array}$ & $\begin{array}{l}\text { MDS } \\
\text { with SNV }\end{array}$ & $\begin{array}{l}\text { MDS } \\
\text { without SNV }\end{array}$ & $\begin{array}{l}\text { Fisher } \\
\mathrm{p} \text { value }\end{array}$ \\
\hline chr13.tRNA1-PheGAA & No & 13 & 95201919 & T & A & 0 & 11 & 4 & 3 & 0.01 \\
\hline chr12.tRNA11-PheGAA & No & 12 & 125412404 & $\mathrm{~T}$ & A & 0 & 16 & 4 & 6 & 0.01 \\
\hline chr6.tRNA44-SerAGA & No & 6 & 27446616 & G & A & 0 & 21 & 5 & 13 & 0.01 \\
\hline chr6.tRNA46-SerAGA & No & 6 & 27463618 & G & A & 0 & 21 & 5 & 13 & 0.01 \\
\hline chr6.tRNA47-SerAGA & No & 6 & 27470843 & G & A & 0 & 21 & 5 & 13 & 0.01 \\
\hline chr6.tRNA50-SerAGA & No & 6 & 27500012 & G & A & 0 & 21 & 5 & 13 & 0.01 \\
\hline chr6.tRNA51-SerTGA & No & 6 & 27513493 & G & A & 0 & 21 & 5 & 13 & 0.01 \\
\hline chr6.tRNA5-SerAGA & No & 6 & 26327842 & G & A & 0 & 21 & 5 & 14 & 0.02 \\
\hline chr6.tRNA148-SerTGA & No & 6 & 27473663 & C & $\mathrm{T}$ & 0 & 21 & 5 & 14 & 0.02 \\
\hline chr6.tRNA147-SerAGA & No & 6 & 27509610 & C & $\mathrm{T}$ & 0 & 21 & 5 & 14 & 0.02 \\
\hline chr17.tRNA35-SerAGA & No & 17 & 8129984 & C & $\mathrm{T}$ & 0 & 21 & 5 & 14 & 0.02 \\
\hline chr6.tRNA172-SerTGA & No & 6 & 26312880 & C & $\mathrm{T}$ & 0 & 21 & 5 & 15 & 0.02 \\
\hline chr7.tRNA21-CysGCA & rs192094984 & 7 & 149112285 & G & A & 0 & 16 & 3 & 7 & 0.05 \\
\hline chr8.tRNA11-SerAGA & No & 8 & 96281941 & C & $\mathrm{T}$ & 0 & 21 & 5 & 17 & 0.05 \\
\hline
\end{tabular}

MDS from controls. MT-TS1 was the only tRNA from this subset that had significant association with treatment response.

Through SNV analysis, we identified 14 SNVs that have potential associations with MDS (Table 4). These 14 SNVs were found in at least $20 \%$ of MDS samples and were not observed in any of the control samples. Only one out of the 14 SNVs has been previously identified (rs192094984) by the 1000 Genomes Project. Some of the SNVs were identified in the same tRNA with the same anticodon but were located at different genomic positions. It is possible that they are the same SNV duplicated by alignment ambiguity.

\section{Discussion}

In this study, using miRNA sequencing data generated from 69 BM-MNC samples and a novel bioinformatics approach, we demonstrated that tDRs are a major byproduct of miRNA sequencing, exceeding the abundance of miRNAs detected by this methodology by nearly 18 -fold. This finding demonstrates the difficulties inherent in studying low-abundance RNA species by expression analysis and emphasizes the importance of adequate filtration.

Due to the potential information embodied in the tDR dataset and a lack of published data on the topic, we chose to study the expression of tDRs in MDS. We demonstrated that six tDRs are differentially found in MDS and control samples, and that a pattern of tRNA species could be used to differentiate MDS and control samples using unsupervised hierarchical clustering. Most significantly, the expression of tDRs in pre-treatment samples was found to predict response to treatment with DNMTIs. These tDRs were found to explain $67 \%$ of the variation in treatment response. The expression of these entities could be used in the clinical setting to select patients likely to respond to DNMTIs. Since only a minority of patients achieve a clinically meaningful response of hematologic improvement, partial response, or complete response to DNMTI therapy, and since it typically takes 3-6 cycles before a response is evident [48], pre-treatment identification of patients likely not to benefit from DNMTI therapy would enable earlier decisions about stem cell transplantation or investigational therapies in those patients. Using changes in tDR expression between pre- and posttreatment samples in model fitting would explain less variation in the data $\left(R^{2}=40 \%\right)$; however, since pre-treatment tRNA expression is a more clinically useful analysis, the better prediction value is fortuitous. Unfortunately, due to the small size of this study, internal validation methods have limited utility. The optimal method to demonstrate the sensitivity and specificity of these biomarkers is to utilize an independent dataset. This will be the focus of future efforts.

Due to the recent developments in the study of mttRNAs and their association with various human diseases [60], the intriguing studies on mitochondrial aberrations in MDS [73], and the identification of chrM.tRNA12.TS1 and chrM.tRNA10.TC in our initial analyses, we examined the expression of mt-tRNA-derived species in MDS. We identified $22 \mathrm{mt}$-tDRs which individually are differentially expressed in MDS compared to control samples, as well as in responders and non-responders to DNMTI therapy. Again, these may be valuable diagnostic and prognostic markers in MDS; however, the correlation between this functional subset tDRs and the mitochondrial aberrations seen in MDS is unclear. 
Finally, we found increased SNVs within the tRNA sequences in the MDS samples as compared to the controls. While this may simply point to underlying genomic instability of myeloid neoplasms, studies in acute myeloid leukemia have not generally found increased absolute numbers of mutations [74], and many mutations are found as a result of aging alone in individuals without any significant hematolymphoid malignancy [75-77]. Therefore, there may be real functional consequences of these SNVs which may affect the functions of these tRNAderived species. It should be noted that RNA-seq is not a well-accepted method for the identification of SNVs due to the high error rate of the reverse transcription step and the RNA editing process $[78,79]$. In addition, the presence of numerous modified nucleotides in tRNAs (e.g. methylation) can result in base errors during the reverse transcription step of RNA-seq [22]. However, these SNVs were not identified to an appreciable level in the control samples, which were processed identically in a blinded fashion, suggesting that the significance of this finding is not an artifact.

Study of tRNA fragments using miRNA sequencing data is not without limitation. As we described previously, tRNAs are typically 73-94 nucleotides in length, composed of three hairpin-turns in a cloverleaf twodimensional structure. The most 5 ' of these loops is the D loop. Within this loop there is a site that has been shown to be sensitive to cleavage either by Dicer or by simple incubation with $\mathrm{MgCl}_{2}$ to afford a 19 nucleotide product from the 5 ' end [10]. However, figures in this same publication also identify a cleavage product of greater than 30 nucleotides [10] that is too small to correspond to the residual 3' fragment. As we have shown, 33 nucleotide tRNA derivatives were identified by our sequencing efforts, resulting from cleavage within the second hairpin loop containing the anticodon. Our size selection step would have excluded most of the 19 nucleotide product. In addition, cleavage in the anti-codon loop occurs physiologically as a result of cleavage by angiogenin [18] and may be targeted by other nucleases as well [19]. Lastly, due to the poor reverse transcription of full length parent tRNAs due to nucleotide modifications, apparent tDRs may result from incomplete reverse transcription [23]. Thus, for various biological and technical reasons, it is possible that under the conditions of this study, the 33 base derivative may be the predominant 5 ' tDR product.

The lack of knowledge of the entire sequence of the tRNA creates ambiguous tRNA annotation. Many of the tRNA isotypes have not only the same or similar anticodons, but also highly homologous sequences 5 ' to the anticodon. This creates challenges in the identification of specific species that may be diagnostically or prognostically useful. As mentioned in the results, several of the prognostic tRNA fragments are homologous. Therefore, although the degenerate species are individually identified, the prognostic power may lie in only one of the entities. Also, in Table 4, a G>A SNV was identified in five SerAGA tRNAs. Since the first 33 base pairs of these five tRNAs are the same, the redundant alignment resulted in the identification of a G>A SNV in all five SerAGA tRNAs for the homologous position, when in fact it may have only been seen in as few as one of the five SerAGA isotypes. The actual nucleotide sequences of each of the tDRs specifically mentioned in this study are available for comparison in Additional file 1: Table S5. Furthermore, we used VarScan 2 to identify SNVs. Typically, at least $20 \%$ of the reads need to support the alternative allele in order to be called an SNV by VarScan 2. So, although none of the control samples in Table 4 contained an SNV as determined by VarScan 2, there may have been low levels of reads that did not meet the $20 \%$ threshold for identification of an SNV.

It is unclear if the expression of tDRs represents the true expression of the full-length tRNAs themselves, if they are physiologic by-products whose presence at different levels suggests differential processing and, therefore, half-lives of the tRNAs, or if they represent biologically active entities in themselves. Needless to say, this ambiguity complicates the further exploration of these results, requiring novel methods to unravel this unknown biology. Recently, new approaches to library preparation have emerged, including the use of demethylases and other strategies to remove modifications prior to reverse transcription. Moreover, the use of Group II intron reverse transcriptases, that bind more tightly than retroviral enzymes to the RNA template, has been demonstrated to overcome modification barriers to afford full-length tRNA cDNAs in RNA-seq libraries [80-83]. These methods may be useful in the future to distinguish the biological tDRs from those that result from technical artifact.

The exact mechanism by which tDRs may play a role in the diagnosis of MDS and prognostication of response to DNMTIs is unclear. There are multitudinous effects of global hypomethylation that results from DNMTI therapy. Some tDRs have been demonstrated to function independently as biologically active entities. Lee et al. have identified small tRNA-derived species, including tRF1001, which promotes the G2/M transition [11]. However, the species identified by Lee et al. are typically 17-26 bases long, smaller than the 33 nucleotide reads identified in this study. Several reports suggest that 30-35 nucleotide long tRNA-derived fragments may play a role in the biogenesis of other small RNAs, and these have been found in wide ranges of species, including humans. Yamasaki et al. suggest that these fragments may mediate stress-related translational repression [84]. However, these explanations would have unclear biologic importance in 
the differential expression of specific tRNA-derived sequences. Alterations in the activity of DICER, and hence of miRNAs, are a common feature in cancers [85-87]. tRNAs have been proposed as alternative DICER1 substrates [39]. Whether certain tRNAs are selectively targeted by DICER1 in cancer in general, and MDS in particular, remains to be seen, but it provides an intriguing hypothesis for the differentially expressed tDRs identified in this study in association with both the diagnosis and prognosis of MDS.

\section{Conclusions}

This study suggests that tRNA-derived fragment sequencing can provide an additional source of data that potentially provides clinically useful applications in diagnosis and prognosis of disease. In addition, this study raises intriguing questions about the biology of these tRNA derivatives in MDS and other cancers.

\section{Additional files}

Additional file 1: Table S1. Sample description. Table S2. Phenotype and alignment results for all samples. Table S3. Differential expression analysis between MDS and control samples. Table S4. Differential expression analysis between pre and post treatment. Table S5.

Nucleotide sequences of the tRNAs described in this paper. (ODS $152 \mathrm{~kb}$ )

Additional file 2: Figure S1. Using a single sample as an example, this figure shows the alignment position of each tRNA. The majority of the alignment lies between residues 1-33 from the $5^{\prime}$ end. The size of each dot indicates the abundance of reads aligned to this position. (PNG $21 \mathrm{~kb}$ )

\begin{abstract}
Abbreviations
miRNA: microRNA; miRNA-seq: microRNA sequencing; MDS: Myelodysplastic syndromes; tRNA: Transfer RNA; tRHs: Transfer RNA halves; tDRs: Transfer RNA-derived small RNAs; nt: Nucleotide; AML: Acute myeloid leukemia; DNMTIs: DNA methyltransferase inhibitors; BM-MNCs: Bone marrow mononuclear cells; totRNA: Total RNA; SNV: Single nucleotide variant; $\mathrm{CV}$ : Cross-validation; mt-tRNAs: Mitochondrial transfer RNAs; FDR: False discovery rate.
\end{abstract}

\section{Competing interests}

The authors declare that they have no competing interests related to this research.

\section{Authors' contributions}

YG and ASK contributed to the conception and design of the study. AB, SM $B E, M J, S A S, E A G$, and $A S K$ were involved in the acquisition of the data. YG, $A B, S M, Q S, F Y, C-I L, P F S, S Z$, and ASK were involved in the analysis and interpretation of the data. $Y G, A B, F Y, K C V$, and $A S K$ were involved in the drafting of the manuscript and critical revisions for important intellectual content. All authors have given final approval of the version to be published. YG and ASK agree to be accountable for all aspects of the work.

\section{Authors' information}

Not applicable.

\section{Availability of data and materials}

Not applicable.

\section{Acknowledgement}

We would like to thank Margot Bjoring and Page Hoskins for editorial support.

\section{Funding}

Yan Guo was supported by NIH Cancer Center Support Grant (P30 CA068485). Annette S. Kim was supported by Doris Duke Charitable Foundation, Clinical Scientist Development Award (\#112076). Sequencing was performed at the Vanderbilt Technologies for Advanced Genomics, which is supported by NIH Cancer Center Support Grant (5P30 CA068485).

\section{Author details}

'Department of Cancer Biology, Vanderbilt University Medical Center, Nashville, TN, USA. ²Department of Pathology, Immunology, and Microbiology, Vanderbilt University Medical Center, Nashville, TN, USA. ${ }^{3}$ Department of Biostatistics, Vanderbilt University, Nashville, TN, USA. ${ }^{4}$ Department of Medicine, Vanderbilt University Medical Center, Nashville, TN, USA. ${ }^{5}$ Department of Applied Mathematics, National Chiayi University, Chiayi City, Taiwan. ${ }^{6}$ Department of Statistics, National Cheng Kung University, Tainan City, Taiwan. ${ }^{7}$ Roswell Park Cancer Institute, Elm \& Carlton Sts, Buffalo, NY, USA. ${ }^{8}$ Present address: Brigham and Women's Hospital, 75 Francis Street, Boston, MA 02115, USA.

Received: 27 May 2015 Accepted: 16 September 2015

Published online: 24 September 2015

\section{References}

1. Wang Z, Gerstein M, Snyder M. RNA-Seq: a revolutionary tool for transcriptomics. Nat Rev Genet. 2009;10(1):57-63.

2. Shendure J. The beginning of the end for microarrays? Nat Methods 2008;5(7):585-7.

3. Guo Y, Sheng Q, Li J, Ye F, Samuels DC, Shyr Y. Large Scale Comparison of Gene Expression Levels by Microarrays and RNAseq Using TCGA Data. PLoS One. 2013;8(8):e71462.

4. Guo Y, Li Cl, Ye F, Shyr Y. Evaluation of read count based RNAseq analysis methods. BMC Genomics. 2013;14 Suppl 8:S2.

5. Asmann YW, Klee EW, Thompson EA, Perez EA, Middha S, Oberg AL, et al. $3^{\prime}$ tag digital gene expression profiling of human brain and universal reference RNA using Illumina Genome Analyzer. BMC Genomics. 2009;10:531

6. Cloonan N, Forrest AR, Kolle G, Gardiner BB, Faulkner GJ, Brown MK, et al. Stem cell transcriptome profiling via massive-scale mRNA sequencing. Nat Methods. 2008;5(7):613-9.

7. Marioni JC, Mason CE, Mane SM, Stephens M, Gilad Y. RNA-seq: an assessment of technical reproducibility and comparison with gene expression arrays. Genome Res. 2008;18(9):1509-17.

8. Martens-Uzunova ES, Olvedy M, Jenster G. Beyond microRNA - Novel RNAs derived from small non-coding RNA and their implication in cancer. Cancer Lett. 2013;340:201-11.

9. Kawaji H, Nakamura M, Takahashi Y, Sandelin A, Katayama S, Fukuda S, et al. Hidden layers of human small RNAs. BMC Genomics. 2008;9(1):157.

10. Cole C, Sobala A, Lu C, Thatcher SR, Bowman A, Brown JW, et al. Filtering of deep sequencing data reveals the existence of abundant Dicer-dependent small RNAs derived from tRNAs. RNA. 2009;15(12):2147-60.

11. Lee YS, Shibata Y, Malhotra A, Dutta A. A novel class of small RNAs: tRNAderived RNA fragments (tRFs). Genes Dev. 2009;23(22):2639-49.

12. Thompson DM, Parker R. Stressing out over tRNA cleavage. Cell. 2009;138(2):215-9.

13. Guo Y, Bosompem A, Zhong X, Clark T, Shyr Y, Kim AS. A comparison of microRNA sequencing reproducibility and noise reduction using mirVana and TRIzol isolation methods. Int J Comput Biol Drug Des. 2014;7(2-3):102-12.

14. Han L, Vickers KC, Samuels DC, Guo Y. Alternative applications for distinct RNA sequencing strategies. Brief Bioinform. 2014;16:629-39.

15. Vickers KC, Roteta LA, Hucheson-Dilks H, Han L, Guo Y. Mining diverse small RNA species in the deep transcriptome. Trends Biochem Sci. 2015;40(1):4-7.

16. Liao JY, Ma LM, Guo YH, Zhang YC, Zhou H, Shao P, et al. Deep sequencing of human nuclear and cytoplasmic small RNAs reveals an unexpectedly complex subcellular distribution of miRNAs and tRNA 3' trailers. PLoS One. 2010;5(5):e10563.

17. Garcia-Silva MR, Cabrera-Cabrera F, Guida MC, Cayota A. Hints of tRNADerived Small RNAs Role in RNA Silencing Mechanisms. Genes (Basel). 2012;3(4):603-14

18. Fu H, Feng J, Liu Q, Sun F, Tie Y, Zhu J, et al. Stress induces tRNA cleavage by angiogenin in mammalian cells. FEBS Lett. 2009;583(2):437-42. 
19. Harada F, Dahlberg JE. Specific cleavage of tRNA by nuclease S1. Nucleic Acids Res. 1975;2(6):865-71.

20. Agris PF. The importance of being modified: an unrealized code to RNA structure and function. RNA. 2015;21(4):552-4.

21. Woodson SA, Muller JG, Burrows CJ, Rokita SE. A primer extension assay for modification of quanine by Ni(II) complexes. Nucleic Acids Res. 1993;21(23):5524-5

22. Ryvkin P, Leung YY, Silverman IM, Childress M, Valladares O, Dragomir I, et al. HAMR: high-throughput annotation of modified ribonucleotides. Rna-a Publication of the Rna Society. 2013:19(12):1684-92.

23. Jackman JE, Montange RK, Malik HS, Phizicky EM. Identification of the yeast gene encoding the tRNA M1G methyltransferase responsible for modification at position 9. RNA. 2003;9(5):574-85.

24. Alazami AM, Hijazi H, Al-Dosari MS, Shaheen R, Hashem A, Aldahmesh MA, et al. Mutation in ADAT3, encoding adenosine deaminase acting on transfer RNA, causes intellectual disability and strabismus. J Med Genet. 2013;50(7):425-30

25. Abbasi-Moheb L, Mertel S, Gonsior M, Nouri-Vahid L, Kahrizi K, Cirak S, et al. Mutations in NSUN2 cause autosomal-recessive intellectual disability. Am J Hum Genet. 2012;90(5):847-55.

26. Najmabadi H, Hu H, Garshasbi M, Zemojtel T, Abedini SS, Chen W, et al. Deep sequencing reveals 50 novel genes for recessive cognitive disorders. Nature. 2011:478(7367):57-63.

27. Frye M, Watt FM. The RNA methyltransferase Misu (NSun2) mediates Mycinduced proliferation and is upregulated in tumors. Curr Biol. 2006;16(10):971-81.

28. Berg M, Agesen TH, Thiis-Evensen E, Merok MA, Teixeira MR, Vatn MH, et al. Distinct high resolution genome profiles of early onset and late onset colorectal cancer integrated with gene expression data identify candidate susceptibility loci. Mol Cancer. 2010;9:100.

29. Bartlett JM, Thomas J, Ross DT, Seitz RS, Ring BZ, Beck RA, et al. Mammostrat as a tool to stratify breast cancer patients at risk of recurrence during endocrine therapy. Breast Cancer Res. 2010;12(4):R47.

30. Wei FY, Suzuki T, Watanabe S, Kimura S, Kaitsuka T, Fujimura A, et al. Deficit of tRNA(Lys) modification by Cdkal1 causes the development of type 2 diabetes in mice. J Clin Invest. 2011;121(9):3598-608.

31. Kirchhoff K, Machicao F, Haupt A, Schafer SA, Tschritter O, Staiger $\mathrm{H}$, et al. Polymorphisms in the TCF7L2, CDKAL1 and SLC30A8 genes are associated with impaired proinsulin conversion. Diabetologia. 2008:51(4):597-601.

32. Omori S, Tanaka Y, Takahashi A, Hirose H, Kashiwagi A, Kaku K, et al. Association of CDKAL1, IGF2BP2, CDKN2A/B, HHEX, SLC3OA8, and KCNJ11 with susceptibility to type 2 diabetes in a Japanese population. Diabetes. 2008:57(3):791-5.

33. Yasukawa T, Suzuki T, Ueda T, Ohta S, Watanabe K. Modification defect at anticodon wobble nucleotide of mitochondrial tRNAs(Leu) (UUR) with pathogenic mutations of mitochondrial myopathy, encephalopathy, lactic acidosis, and stroke-like episodes. J Biol Chem. 2000;275(6):4251-7.

34. Yasukawa T, Kirino Y, Ishii N, Holt IJ, Jacobs HT, Makifuchi T, et al. Wobble modification deficiency in mutant tRNAs in patients with mitochondrial diseases. FEBS Lett. 2005;579(13):2948-52.

35. Kirino Y, Goto Y, Campos Y, Arenas J, Suzuki T. Specific correlation between the wobble modification deficiency in mutant tRNAs and the clinical features of a human mitochondrial disease. Proc Natl Acad Sci U S A. 2005;102(20):7127-32.

36. Gattermann N, Wulfert M, Junge B, Germing U, Haas R, Hofhaus G. Ineffective hematopoiesis linked with a mitochondrial tRNA mutation (G3242A) in a patient with myelodysplastic syndrome. Blood. 2004;103(4):1499-502

37. Erdogan B, Bosompem A, Peng D, Han L, Smith E, Kennedy ME, et al. Methylation of promoters of microRNAs and their host genes in myelodysplastic syndromes. Leukemia \& lymphoma. 2013;54:2720-7.

38. Erdogan B, Facey C, Qualtieri J, Tedesco J, Rinker E, Isett RB, et al. Diagnostic microRNAs in myelodysplastic syndrome. Exp Hematol. 2011;39(9):915-26:e912.

39. Cogle CR, Craig BM, Rollison DE, List AF. Incidence of the myelodysplastic syndromes using a novel claims-based algorithm: high number of uncaptured cases by cancer registries. Blood. 2011;117(26):7121-5.

40. Ebert BL, Pretz J, Bosco J, Chang CY, Tamayo P, Galili N, et al. Identification of RPS14 as a 5q- syndrome gene by RNA interference screen. Nature. 2008:451(7176):335-9.

41. Starczynowski DT, Kuchenbauer F, Argiropoulos B, Sung S, Morin R, Muranyi A, et al. Identification of miR-145 and miR-146a as mediators of the 5qsyndrome phenotype. Nat Med. 2010;16(1):49-58.
42. Dostalova Merkerova M, Krejcik Z, Votavova H, Belickova M, Vasikova A, Cermak J. Distinctive microRNA expression profiles in CD34+ bone marrow cells from patients with myelodysplastic syndrome. Eur J Hum Genet. 2011;19(3):313-9.

43. Hussein K, Theophile K, Busche G, Schlegelberger B, Gohring G, Kreipe H, et al. Aberrant microRNA expression pattern in myelodysplastic bone marrow cells. Leuk Res. 2010;34(9):1169-74.

44. Pons A, Nomdedeu B, Navarro A, Gaya A, Gel B, Diaz T, et al. Hematopoiesisrelated microRNA expression in myelodysplastic syndromes. Leuk Lymphoma. 2009;50:1854-9.

45. Sokol L, Caceres G, Volinia S, Alder H, Nuovo GJ, Liu CG, et al. Identification of a risk dependent microRNA expression signature in myelodysplastic syndromes. Br J Haematol. 2011;153(1):24-32.

46. Ma X, Does M, Raza A, Mayne ST. Myelodysplastic syndromes: incidence and survival in the United States. Cancer. 2007;109(8):1536-42.

47. Swerdlow SH, Campo E, Harris NL, Jaffe ES, Pileri SA, Stein H, et al. World Health Organization Classification of Tumours of Haematopoietic and Lymphoid Tissues. 4th ed. Lyon, France: IARC Press; 2008.

48. Silverman LR, McKenzie DR, Peterson BL, Holland JF, Backstrom JT, Beach CL, et al. Further analysis of trials with azacitidine in patients with myelodysplastic syndrome: studies 8421,8921 , and 9221 by the Cancer and Leukemia Group B. J Clin Oncol. 2006;24(24):3895-903.

49. Bejar R, Lord A, Stevenson K, Bar-Natan M, Perez-Ladaga A, Zaneveld J, et al. TET2 mutations predict response to hypomethylating agents in myelodysplastic syndrome patients. Blood. 2014;124(17):2705-12.

50. Abaigar M, Ramos F, Benito R, Diez-Campelo M, Sanchez-del-Real J, Hermosin $\mathrm{L}$, et al. Prognostic impact of the number of methylated genes in myelodysplastic syndromes and acute myeloid leukemias treated with azacytidine. Ann Hematol. 2013;92(11):1543-52

51. Valencia A, Masala E, Rossi A, Martino A, Sanna A, Buchi F, et al. Expression of nucleoside-metabolizing enzymes in myelodysplastic syndromes and modulation of response to azacitidine. Leukemia. 2014;28(3):621-8.

52. Figueroa ME, Skrabanek L, Li Y, Jiemjit A, Fandy TE, Paietta E, et al. MDS and secondary AML display unique patterns and abundance of aberrant DNA methylation. Blood. 2009;114(16):3448-58.

53. Flotho C, Claus R, Batz C, Schneider M, Sandrock I, Ihde S, et al. The DNA methyltransferase inhibitors azacitidine, decitabine and zebularine exert differential effects on cancer gene expression in acute myeloid leukemia cells. Leukemia. 2009;23(6):1019-28.

54. Mund C, Hackanson B, Stresemann C, Lubbert M, Lyko F. Characterization of DNA demethylation effects induced by 5-Aza-2'-deoxycytidine in patients with myelodysplastic syndrome. Cancer Res. 2005;65(16):7086-90.

55. Meldi K, Qin T, Buchi F, Droin N, Sotzen J, Micol JB, et al. Specific molecular signatures predict decitabine response in chronic myelomonocytic leukemia. J Clin Invest. 2015;125(5):1857-72.

56. Cheson BD, Greenberg PL, Bennett JM, Lowenberg B, Wijermans PW, Nimer SD, et al. Clinical application and proposal for modification of the International Working Group (IWG) response criteria in myelodysplasia. Blood. 2006;108(2):419-25.

57. Martin M. Cutadapt removes adapter sequences from high-throughput sequencing reads. EMBnetjournal. 2011;17:10-2.

58. Guo Y, Zhao S, Sheng Q, Ye F, Li J, Lehmann B, et al. Multi-perspective quality control of Illumina exome sequencing data using QC3. Genomics. 2014;103:323-8.

59. Langmead B, Trapnell C, Pop M, Salzberg SL. Ultrafast and memory-efficient alignment of short DNA sequences to the human genome. Genome Biol. 2009;10(3):R25

60. Kozomara A, Griffiths-Jones S. miRBase: annotating high confidence microRNAs using deep sequencing data. Nucleic Acids Res. 2014:42(Database issue):D68-73.

61. Flicek P, Amode MR, Barrell D, Beal K, Billis K, Brent S, et al. Ensembl 2014 Nucleic Acids Res. 2014:42(Database issue):D749-55.

62. Miller AC, Obholzer ND, Shah AN, Megason SG, Moens CB. RNA-seq-based mapping and candidate identification of mutations from forward genetic screens. Genome Res. 2013;23(4):679-86.

63. Duitama J, Srivastava P, Mandoiu I. Towards accurate detection and genotyping of expressed variants from whole transcriptome sequencing data. BMC Genomics. 2012;13 Suppl 2:S6.

64. Piskol R, Ramaswami G, Li JB. Reliable Identification of Genomic Variants from RNA-Seq Data. Am J Hum Genet. 2013;93(4):641-51.

65. Koboldt DC, Zhang $\mathrm{Q}$, Larson $\mathrm{DE}$, Shen $\mathrm{D}$, McLellan MD, Lin L, et al. VarScan 2: somatic mutation and copy number alteration discovery in cancer by exome sequencing. Genome Res. 2012;22(3):568-76. 
66. Guo Y, Zhao S, Ye F, Sheng Q, Shyr Y. MultiRankSeq: Multiperspective Approach for RNAseq Differential Expression Analysis and Quality Control. BioMed Res International. 2014;2014:8.

67. Anders $\mathrm{S}$, Huber W. Differential expression analysis for sequence count data. Genome Biol. 2010;11(10):R106.

68. Robinson MD, McCarthy DJ, Smyth GK. edgeR: a Bioconductor package for differential expression analysis of digital gene expression data. Bioinformatics. 2010;26(1):139-40.

69. Hardcastle TJ, Kelly KA. baySeq: empirical Bayesian methods for identifying differential expression in sequence count data. BMC Bioinformatics. 2010;11:422

70. Zou H, Hastie T. Regularization and variable selection via the elastic net (vol B 67, pg 301, 2005). J Royal Stat Soc Series B-Stat Method. 2005;67:768.

71. Tibshirani R. Regression shrinkage and selection via the Lasso. J Royal Stat Soc Series B-Stat Method. 1996;58(1):267-88.

72. Zhao S, Guo Y, Sheng Q, Shyr Y. Advanced Heat Map and Clustering Analysis Using Heatmap3. BioMed Research International. 2014;2014:6.

73. Chen ML, Logan TD, Hochberg ML, Shelat SG, Yu X, Wilding GE, et al. Erythroid dysplasia, megaloblastic anemia, and impaired lymphopoiesis arising from mitochondrial dysfunction. Blood. 2009;114(19):4045-53.

74. Welch JS, Ley TJ, Link DC, Miller CA, Larson DE, Koboldt DC, et al. The origin and evolution of mutations in acute myeloid leukemia. Cell. 2012:150(2):264-78.

75. Genovese G, Kahler AK, Handsaker RE, Lindberg J, Rose SA, Bakhoum SF, et al. Clonal hematopoiesis and blood-cancer risk inferred from blood DNA sequence. N Engl J Med. 2014;371(26):2477-87.

76. Jaiswal S, Fontanillas P, Flannick J, Manning A, Grauman PV, Mar BG, et al. Age-related clonal hematopoiesis associated with adverse outcomes. N Engl J Med. 2014;371(26):2488-98.

77. Xie M, Lu C, Wang J, McLellan MD, Johnson KJ, Wendl MC, et al. Age-related mutations associated with clonal hematopoietic expansion and malignancies. Nat Med. 2014;20(12):1472-8.

78. Roberts JD, Bebenek K, Kunkel TA. The accuracy of reverse transcriptase from HIV-1. Science. 1988;242(4882):1171-3.

79. O'Brien TD, Jia P, Xia J, Saxena U, Jin H, Vuong H, et al. Inconsistency and features of single nucleotide variants detected in whole exome sequencing versus transcriptome sequencing: A case study in lung cancer. Methods. 2015;83:118-27.

80. Mohr S, Ghanem E, Smith W, Sheeter D, Qin Y, King O, et al. Thermostable group II intron reverse transcriptase fusion proteins and their use in cDNA synthesis and next-generation RNA sequencing. RNA. 2013;19(7):958-70.

81. Enyeart PJ, Mohr G, Ellington AD, Lambowitz AM. Biotechnological applications of mobile group II introns and their reverse transcriptases: gene targeting, RNA-seq, and non-coding RNA analysis. Mob DNA. 2014;5:2

82. Shen PS, Park J, Qin YD, Li XM, Parsawar K, Larson MH, et al. Rqc2p and 60S ribosomal subunits mediate mRNA-independent elongation of nascent chains. Science. 2015;347(6217):75-8.

83. Katibah GE, Qin YD, Sidote DJ, Yao J, Lambowitz AM, Collins K. Broad and adaptable RNA structure recognition by the human interferon-induced tetratricopeptide repeat protein IFIT5. Proc Natl Acad Sci U S A. 2014;111(33):12025-30.

84. Yamasaki S, Ivanov P, Hu GF, Anderson P. Angiogenin cleaves tRNA and promotes stress-induced translational repression. J Cell Biol. 2009;185(1):35-42.

85. Adams CM, Eischen CM. Inactivation of p53 Is Insufficient to Allow B Cells and B-Cell Lymphomas to Survive Without Dicer. Cancer Res. 2014;74(14):3923-34.

86. Anglesio MS, Wang Y, Yang W, Senz J, Wan A, Heravi-Moussavi A, et al. Cancer-associated somatic DICER1 hotspot mutations cause defective miRNA processing and reverse-strand expression bias to predominantly mature $3 p$ strands through loss of $5 p$ strand cleavage. J Pathol. 2013;229(3):400-9.

87. Heravi-Moussavi A, Anglesio MS, Cheng SW, Senz J, Yang W, Prentice L, et al. Recurrent somatic DICER1 mutations in nonepithelial ovarian cancers. N Engl J Med. 2012;366(3):234-42.

88. Zeviani M, Muntoni F, Savarese N, Serra G, Tiranti V, Carrara F, et al. A MERRF/MELAS overlap syndrome associated with a new point mutation in the mitochondrial DNA tRNA(Lys) gene. Eur J Hum Genet. 1993;1(1):80-7.

89. Thorburn DR, Rahman S. Mitochondrial DNA-Associated Leigh Syndrome and NARP. Seattle: University of Washington; 1993.
90. Mahjoub S, Sternberg D, Boussaada R, Filaut S, Gmira F, Mechmech R, et al. A novel mitochondrial DNA tRNAlle (m.4322dupC) mutation associated with idiopathic dilated cardiomyopathy. Diagn Mol Pathol. 2007;16(4):238-42.

91. Bataillard M, Chatzoglou E, Rumbach L, Sternberg D, Tournade A, Laforet P, et al. Atypical MELAS syndrome associated with a new mitochondrial tRNA glutamine point mutation. Neurology. 2001;56(3):405-7.

92. Vissing J, Salamon MB, Arlien-Soborg P, Norby S, Manta P, DiMauro S, et al. A new mitochondrial tRNA(Met) gene mutation in a patient with dystrophic muscle and exercise intolerance. Neurology. 1998;50(6):1875-8.

93. del Mar O'CM, Emperador S, Lopez-Gallardo E, Jou C, Bujan N, Montero R, et al. New mitochondrial DNA mutations in tRNA associated with three severe encephalopamyopathic phenotypes: neonatal, infantile, and childhood onset. Neurogenetics. 2012;13(3):245-50.

94. Tang XW, Li RH, Zheng J, Cai Q, Zhang T, Gong SS, et al. Maternally inherited hearing loss is associated with the novel mitochondrial tRNA(Ser(UCN)) 7505 T> C mutation in a Han Chinese family. Mol Genet Metab. 2010;100(1):57-64.

95. Seibel P, Lauber J, Klopstock T, Marsac C, Kadenbach B, Reichmann H. Chronic Progressive External Ophthalmoplegia Is Associated with a Novel Mutation in the Mitochondrial Trna(Asn) Gene. Biochem Bioph Res Co. 1994;204(2):482-9.

96. Lehtonen MS, Moilanen JS, Majamaa K. Increased variation in mtDNA in patients with familial sensorineural hearing impairment. Hum Genet. 2003;113(3):220-7.

97. Scaglia F, Vogel H, Hawkins EP, Vladutiu GD, Liu LL, Wong LJ. Novel homoplasmic mutation in the mitochondrial tRNATyr gene associated with atypical mitochondrial cytopathy presenting with focal segmental glomerulosclerosis. Am J Med Genet A. 2003;123A(2):172-8.

98. Levinger $L$, Jacobs $O$, James M. In vitro 3 '-end endonucleolytic processing defect in a human mitochondrial tRNA(Ser(UCN)) precursor with the U7445C substitution, which causes non-syndromic deafness. Nucleic Acids Res. 2001;29(21):4334-40.

99. Seneca S, Goemans N, Van Coster R, Givron P, Reybrouck T, Sciot R, et al. A mitochondrial tRNA aspartate mutation causing isolated mitochondrial myopathy. Am J Med Genet A. 2005;137(2):170-5.

100. Levinger L, Morl M, Florentz C. Mitochondrial tRNA 3 ' end metabolism and human disease. Nucleic Acids Res. 2004;32(18):5430-41.

101. Merante F, Tein I, Benson L, Robinson BH. Maternally Inherited Hypertrophic Cardiomyopathy Due to a Novel T-to-C Transition at Nucleotide-9997 in the Mitochondrial Trna(Glycine) Gene. Am J Hum Genet. 1994;55(3):437-46.

102. Pancrudo J, Shanske S, Coku J, Lu J, Mardach R, Akman O, et al. Mitochondrial myopathy associated with a novel mutation in mtDNA. Neuromuscul Disord. 2007;17(8):651-4.

103. Elson JL, Swalwell H, Blakely EL, McFarland R, Taylor RW, Turnbull DM. Pathogenic Mitochondrial tRNA Mutations - Which Mutations Are Inherited and Why? Hum Mutat. 2009;30(11):E984-92.

104. Wong LUC, Yim D, Bai RK, Kwon H, Vacek MM, Zane J, et al. A novel mutation in the mitochondrial tRNA (Ser(AGY)) gene associated with mitochondrial myopathy, encephalopathy, and complex I deficiency. J Med Genet. 2006;43(9):e46.

105. Zsurka G, Schroder R, Kornblum C, Rudolph J, Wiesner RJ, Elger CE, et al. Tissue dependent co-segregation of the novel pathogenic G12276A mitochondrial tRNALeu(CUN) mutation with the A185G D-loop polymorphism. J Med Genet. 2004;41(12):e124.

106. Mimaki M, Hatakeyama $H$, Komaki H, Yokoyama M, Arai H, Kirino Y, et al. Reversible infantile respiratory chain deficiency: a clinical and molecular study. Ann Neurol. 2010;68(6):845-54.

107. Mayr-Wohlfart U, Paulus C, Henneberg A, Rodel G. Mitochondrial DNA mutations in multiple sclerosis patients with severe optic involvement. Acta Neurol Scand. 1996;94(3):167-71.

108. Wong LJC, Liang MH, Kwon H, Bai RK, Alper O, Gropman A. A cystic fibrosis patient with two novel mutations in mitochondrial DNA: Mild disease led to delayed diagnosis of both disorders. Am J Med Genet. 2002;113(1):59-64. 